\title{
Pathological complete response in breast cancer patients following neoadjuvant chemotherapy at a Comprehensive Cancer Center: The natural history of an elusive prognosticator
}

\author{
OLUWADAMILOLA M. FAYANJU ${ }^{1}$, IHEOMA NWAOGU ${ }^{1}$, DONNA B. JEFFE ${ }^{2,3}$ and JULIE A. MARGENTHALER ${ }^{1,3}$ \\ ${ }^{1}$ Department of Surgery, ${ }^{2}$ Division of General Medical Sciences, Department of Medicine, \\ Washington University School of Medicine; ${ }^{3}$ The Alvin J. Siteman Cancer Center at Barnes Jewish \\ Hospital and Washington University School of Medicine, St. Louis, MO 63110, USA
}

Received January 20, 2015; Accepted March 12, 2015

DOI: $10.3892 / \mathrm{mco} .2015 .535$

\begin{abstract}
Given the prognostic significance of pathological complete response (pCR) to neoadjuvant chemotherapy, we sought to chronicle the clinical course of breast cancer patients whose tumors exhibited pCR at our institution. We retrospectively reviewed 5,533 cancer center patients treated for a first primary breast cancer between March, 1999 and September, 2010 to identify those who received neoadjuvant chemotherapy that resulted in pCR (i.e., no residual invasive malignancy in the breast or axilla). The descriptive statistics of treatments received, recurrence, morbidity and mortality as of October, 2013 were reported. Of the 5,533 patients reviewed, 86 met the inclusion criteria. The mean age at diagnosis was 48 years [standard deviation (SD), 9.4 years] and the mean length of follow-up was 68 months (SD, 27 months). The majority of the patients underwent axillary lymph node dissection (ALND; $\mathrm{n}=60,69.8 \%$ ), received adjuvant radiation therapy (XRT; $\mathrm{n}=72,83.7 \%$ ), had poorly differentiated (grade 3$)$ tumors $(\mathrm{n}=74$, $86.1 \%)$ and had pure ductal histology $(n=74,86.1 \%)$. A total of 5 patients $(5.8 \%)$ developed disease recurrence. All the patients who recurred had grade 3 tumors with ductal histology and underwent ALND for known pre-neoadjuvant-treatment lymph node metastases; none received adjuvant chemotherapy. A total of 4 patients (4.7\%) succumbed to the disease, 3 due to breast cancer recurrence $<18$ months following the initial diagnosis. Recurrence following pCR was rare, but when it did occur, time- to-recurrence was short at $<18$ months. All the patients who recurred and eventually succumbed to breast cancer had axillary metastases at diagnosis, indicating that axillary
\end{abstract}

Correspondence to: Dr Julie A. Margenthaler, The Alvin J. Siteman Cancer Center at Barnes Jewish Hospital and Washington University School of Medicine, 660 S. Euclid Avenue, Campus Box 8109, St. Louis, MO 63110, USA

E-mail: margenthalerj@wudosis.wustl.edu

Key words: breast cancer, neoadjuvant chemotherapy, pathological complete response disease is a major negative prognostic factor in patients who achieve pCR following neoadjuvant chemotherapy.

\section{Introduction}

As our knowledge of the human genome and the molecular subtypes of particular cancers increases, the field of breast oncology is becoming increasingly focused on individualizing treatment based on the clinical and biochemical profile of a given patient. Nomograms are being developed and used to guide the type and extent of therapy patients receive, with the hope that we optimally treat many and overtreat few $(1,2)$. As we collectively navigate the path from clinical assessment to individualized treatment to optimized outcome, the significance of proximal clinical endpoints must not be underestimated, as they assist clinicians in determining whether to proceed with or modify a given patient's treatment course and may also facilitate pharmaceutical development by providing a surrogate endpoint of efficacy that does not mandate the long follow-up periods required to evaluate differences in survival or recurrence $(3,4)$.

Pathological complete response (pCR) to neoadjuvant chemotherapy has emerged as a reliable prognostic indicator for overall and event-free survival following breast cancer diagnosis (5-10), occurring in $\sim 15-40 \%$ of breast cancer patients receiving neoadjuvant chemotherapy (3). Given the prognostic significance of pCR and the evolution of decision-making nomograms for the management of breast cancer, we aimed to chronicle the clinical course of breast cancer patients at our institution (Siteman Cancer Center, St. Louis, MO, USA) whose index tumors exhibited pCR.

\section{Patients and methods}

Patient selection. Clinical, demographic and pathological data from all the breast cancer patients treated at our institution are prospectively recorded in a database. In a retrospective review of this database, 5,533 patients treated for a first primary breast cancer at Siteman Cancer Center between March, 1999 and September, 2010 were identified. Subsequently, patients who received neoadjuvant chemotherapy for treatment of a 
Table I. Characteristics of breast cancer patients $(n=86)$ exhibiting pCR following neoadjuvant chemotherapy. ${ }^{\mathrm{a}}$

\begin{tabular}{|c|c|}
\hline Clinical characteristics & Values \\
\hline Age, years [median (range)] & $48(27-70)$ \\
\hline Follow-up, months [median (range)] & $39(8-124)$ \\
\hline \multicolumn{2}{|l|}{ Race, n (\%) } \\
\hline African American & $25(29.1)$ \\
\hline Asian American & $1(1.2)$ \\
\hline Caucasian & $60(69.7)$ \\
\hline \multicolumn{2}{|l|}{ Bilaterality, n (\%) } \\
\hline Bilateral & $2(2.3)$ \\
\hline Unilateral & $84(97.7)$ \\
\hline \multicolumn{2}{|l|}{ Lymph node involvement, n (\%) } \\
\hline Yes & $49(57.0)$ \\
\hline No & $37(43.0)$ \\
\hline \multicolumn{2}{|l|}{ TNM stage, n (\%) } \\
\hline Early (I-IIA) & $38(44.2)$ \\
\hline Late (IIB-IIIC) & $48(55.8)$ \\
\hline \multicolumn{2}{|l|}{ Receptor status, n (\%) } \\
\hline \multicolumn{2}{|l|}{ ER } \\
\hline Positive & $25(29.1)$ \\
\hline Negative & $61(70.9)$ \\
\hline \multicolumn{2}{|l|}{ PR } \\
\hline Positive & $17(19.8)$ \\
\hline Negative & $69(80.2)$ \\
\hline \multicolumn{2}{|l|}{ HER2/neu } \\
\hline Positive & $31(36.0)$ \\
\hline Negative & $55(64.0)$ \\
\hline $\mathrm{ER}^{-} / \mathrm{PR}-/ \mathrm{HER} 2^{+}$ & $16(18.6)$ \\
\hline ER-/PR-/HER2- (triple-negative) & $43(50.0)$ \\
\hline
\end{tabular}

Tumor grade, $\mathrm{n}(\%)$

1

2

3

Histology, n (\%)

Ductal only

Lobular only

Other

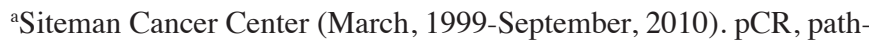
ological complete response; ER, estrogen receptor; PR, progesterone receptor; HER2, human epidermal growth factor receptor 2.

pathologically confirmed invasive (stages I-III) breast cancer were identified and their medical records were reviewed to determine which of these tumors exhibited pCR, defined as no evidence of residual invasive malignancy in the breast or axilla. As ductal carcinoma in situ (DCIS) generally does not regress with chemotherapy and evidence of its effect on prognosis is equivocal $(11,12)$, patients with only residual DCIS following neoadjuvant chemotherapy were included in the pCR cohort.

Institutional Review Board approval was obtained prior to the commencement of this retrospective study at Washington University School of Medicine. Written consent from the patients was not required, due to the retrospective nature of this study.

Statistical analysis. The descriptive statistics of treatments received, recurrence, morbidity and mortality as of October, 2013 are reported. All the statistical analyses were conducted using SAS 9.3 software (SAS Institute Inc., Cary, NC, USA).

\section{Results}

Patient characteristics. Of the 5,533 patients reviewed, 746 received neoadjuvant chemotherapy and 86 (1.6\% of all patients, $11.5 \%$ of neoadjuvant chemotherapy recipients) met the inclusion criteria (Table I). The mean age at diagnosis was 48 years [standard deviation (SD), 9.4 years] and the mean length of follow-up was 68 months (SD, 27 months). The majority of the patients underwent axillary lymph node dissection (ALND; $n=60,69.8 \%$ ), were Caucasian ( $n=60,69.7 \%$ ), received adjuvant radiation therapy (XRT; $n=72,83.7 \%$ ), had poorly differentiated (grade 3 ) tumors $(n=74,86.1 \%)$, had only ductal histology $(n=74,86.1 \%)$ and received a taxane as part of their neoadjuvant treatment $(n=83,96.5 \%)$. Due to inconsistencies in the information documentation process in our database, the administration of anti-human epidermal growth factor receptor 2 (HER2)/neu treatments could not be determined for all patients and, accordingly, summary statistics on this treatment variable were not provided.

Outcome. A total of 5 patients (5.8\%) developed disease recurrence (1 locoregional and distant and 4 distant recurrence) (Table II). The only patient in our cohort with residual DCIS developed recurrence. All the patients who recurred had grade 3 tumors with ductal histology and underwent ALND for known pre-neoadjuvant treatment lymph node metastases; none of the patients with recurrence received adjuvant chemotherapy. A total of 4 patients $(4.7 \%)$ succumbed to the disease, 3 due to breast cancer recurrence $<18$ months after the initial diagnosis and 1 due to metastatic small bowel adenocarcinoma.

\section{Discussion}

High tumor grade and the presence of lymph node metastases at diagnosis were two prominent clinical characteristics shared by all patients who recurred following pCR. High tumor grade has been previously reported as a marker of $\mathrm{pCR}$ probability, as the high mitotic activity of poorly differentiated tumors appears to render them more susceptible to the cytotoxic effects of standard chemotherapy regimens $(13,14)$. However, this potential vulnerability to chemotherapy lies in tension with the fact that high tumor grade has, outside the context of pCR, been demonstrated to be a predictor of breast cancer progression, recurrence and death (15). This paradox points to a greater challenge in therapeutic decision-making for breast cancer patients: Specifically, when trying to determine who is 


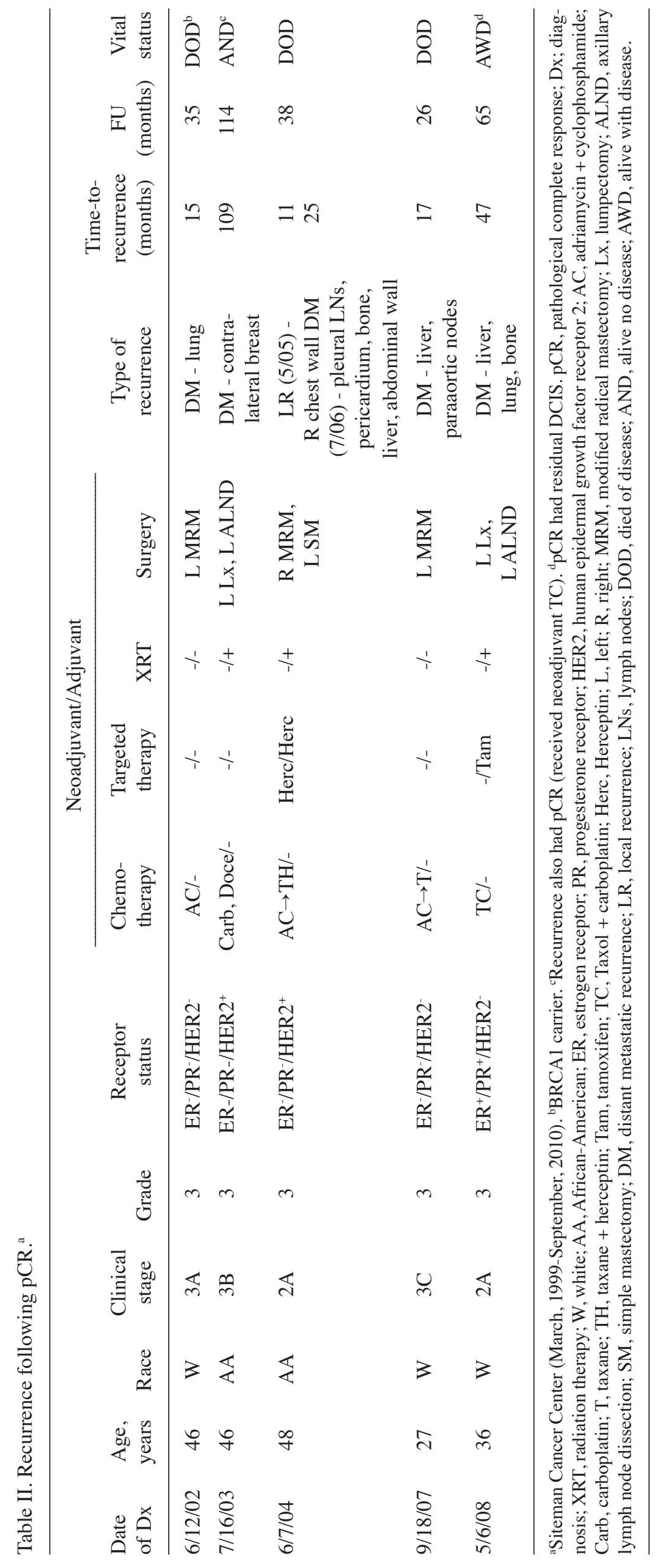


most likely to experience pCR, one needs to consider not only the patients with enhanced susceptibility to neoadjuvant therapies, but also the patients who are most likely to benefit from these treatments. Patients with grade 3 tumors are at the intersection of these two idealized populations: they are more likely to have poor outcomes as a result of their disease, but they are also more likely to respond to chemotherapeutic treatment. Neoadjuvant chemotherapy is generally reserved for patients with locally advanced disease (a number of whom have tumor biology that precludes systemic endocrine therapy) in order to treat disseminated disease and facilitate breast conservation therapy (16). However, a recent study demonstrating the safety of neoadjuvant systemic therapy in early-stage patients (17) should mitigate concerns regarding the administration of preoperative systemic therapy to early-stage patients with poorly differentiated tumors, but displaying other disease characteristics that may otherwise have encouraged oncologists to forego chemotherapy. Thus, administering neoadjuvant chemotherapy to grade 3 patients of all stages may significantly affect the rate of pCR and concomitant outcomes, although additional clinical trials investigating the efficacy of neoadjuvant chemotherapy in early-stage patients are required.

In addition, there is increasing evidence that a certain subset of women with axillary disease at diagnosis require adjuvant systemic therapy, regardless of their local response to neoadjuvant treatment, as demonstrated on operative pathology (18). In our cohort, a total of 5 patients recurred, 3 of whom did so within 18 months of definitive surgical treatment; none of these 5 patients received adjuvant chemotherapy. Such a short interval between diagnosis and post-treatment recurrence likely reflects the failure of neoadjuvant treatment in these patients and is further proof that axillary lymph node metastases should be considered as markers of disseminated disease that may continue to progress regardless of $\mathrm{pCR}$ in the breast and axilla. In addition, all 5 patients with recurrence were aged $<50$ years at diagnosis, young age being a clinical characteristic that compounds the negative prognosticators of high grade and axillary disease at diagnosis.

Finally, recent studies have demonstrated the extent to which the prognostic accuracy of pCR depends on a given tumor's molecular subtype. Specifically, in a large meta-analysis including 7 German randomized trials, von Minckowitz et al found that $\mathrm{pCR}$ appeared to be a reasonable surrogate endpoint for patients with luminal B/HER2-, ER/PR-/HER2 ${ }^{+}$ and triple-negative biomarker profiles, but not for those with luminal B/HER2 ${ }^{+}$or luminal A tumors (12). However, of the 5 patients in our cohort who developed recurrence following $\mathrm{pCR}, 2$ had $\mathrm{ER}^{-} / \mathrm{PR}^{-} / \mathrm{HER} 2^{+}$disease (1 succumbed to the disease and 1 remains alive and disease-free) and 2 had triple-negative disease, both of whom succumbed to their cancer. Thus, even for the subset of patients for whom pCR is a good prognosticator, its accuracy is not $100 \%$.

Major advances have been made over the past 15 years in refining the potential use of pCR with regards to medical decision-making for patients with breast cancer. For example, it was observed that, although tumors with estrogen and/or progesterone receptors are less likely to undergo pCR following neoadjuvant systemic therapy, patients with these tumors tend to have better long-term outcomes compared with patients with hormone receptor-negative disease $(14,16)$. Furthermore,
pCR does not appear to be a useful predictor of outcome for assessing the long-term efficacy of neoadjuvant endocrine therapy, which rarely results in pCR (19). As described above, we are developing a better sense of the optimal way to interpret and utilize pCR in patients with tumor subtypes for whom pCR has been associated with improved outcomes. The next rational step in prognostication with $\mathrm{pCR}$ would be to further delineate which particular molecular subtypes among those predisposed to $\mathrm{pCR}$ are more likely to recur following $\mathrm{pCR}$ and the underlying mechanisms. Notably, the only patient in our cohort who had residual DCIS following neoadjuvant chemotherapy (as allowed for in our inclusion criteria) developed a recurrence. As proposed in a recent study, the standard definition of pCR, in which residual in situ malignancy is allowed to be present, may require revision (12). DCIS may actually have a greater malignant potential than once thought in certain tumor subtypes; in addition, its close proximity to overlooked invasive disease may be of greater significance than previously appreciated (12). None of the patients who recurred received adjuvant therapy, although some may have had both known and unknown clinicopathological characteristics, such as residual DCIS, which may have prompted further treatment had their tumors not shown pCR. For a subset of breast cancer patients who receive neoadjuvant chemotherapy, pCR may confer a false sense of confidence that diverts them and their oncologists away from the systemic adjuvant treatment that may have otherwise prevented recurrence or even saved their lives.

Our findings corroborate previous research demonstrating that, while pCR portends promising outcomes for the majority of neoadjuvant chemotherapy recipients with breast cancer, it holds significantly less prognostic significance for certain women, specifically those whose disease lies at the intersection of multiple negative prognosticators, including high grade, preoperative axillary metastases, young age and aggressive tumor biology.

Additional prospective studies are warranted to determine which clinicopathological characteristics mitigate the prognostic efficacy of pCR as a predictor of disease-free and overall survival among subtypes (namely, ER $/ \mathrm{PR}^{-} / \mathrm{HER}^{+}$ and triple-negative) whose receptor statuses usually lend themselves to accurate prognostication by pCR. Such research would undoubtedly help with the refinement of treatment decision nomograms in women undergoing neoadjuvant chemotherapy.

Our study's findings are important for a number of reasons. First, to the best of our knowledge, the average length of follow-up for our cohort (68 months) is the longest in the literature on pCR in breast cancer, allowing us to demonstrate the relative long-term infrequency of recurrence following pCR, while showing that, when recurrence does occur, it may happen quite soon following pathological confirmation of response. Second, while our study identified characteristics (such as young age and the presence of axillary disease) that were found to be associated with post-pCR recurrence in other larger cohorts $(20,21)$ by drilling down on the pretreatment characteristics, received therapies and post-therapeutic course of our patients who recurred following pCR, we have identified additional factors (including tumor grade, the presence of residual DCIS and non-administration of adjuvant 
chemotherapy) that may warrant additional investigation, both in isolation and in association with each other.

Our study was limited first and foremost by the retrospective nature of our review. Women who receive neoadjuvant chemotherapy are more likely to have hormone receptor-negative, biologically aggressive tumors, for which the availability of alternative treatments is more limited. Accordingly, there is significant selection bias in our sample, which was also insufficient for comparative statistical analysis; we chose not to compare the 86 patients who underwent pCR to the 660 who did not, as we were concerned that uncontrolled differences between these groups would significantly confound attempts at statistical comparison. Second, all the patients in our study were treated at a high-volume NCI-designated Comprehensive Cancer Center, although the majority of the patients in this country obtain their chemotherapy from non-academic, community medical oncologists; thus, the extent to which our patients achieve pCR and the reasons why they do so may not reflect the treatment experience of the typical breast cancer patient.

In conclusion, first primary breast cancer patients whose tumors exhibited pCR following neoadjuvant chemotherapy had low rates of breast cancer-specific mortality and recurrence compared to the general population of breast cancer patients of similar stage, although when recurrence did occur, the time-to-recurrence was often short at $<18$ months. All the patients who recurred and eventually succumbed to breast cancer had poorly differentiated tumors with axillary metastases at initial diagnosis, indicating that axillary lymph node disease burden may itself be a negative prognostic factor in patients with a first primary breast cancer who achieve pCR following neoadjuvant chemotherapy.

\section{Acknowledgements}

The research of Drs Fayanju and Nwaogu was supported by the NIH Ruth L. Kirschstein National Research Service Award Institutional Research Training Grant 5T32CA009621-24. Dr Jeffe was supported in part by the NCI Cancer Center Support Grant P30 CA091842 to the Alvin J. Siteman Cancer Center at Barnes Jewish Hospital and Washington University School of Medicine, St. Louis, MO, USA. Part of this study's findings were presented at the American Society of Breast Surgeons 15th Annual Meeting, April 30-May 4, 2014.

\section{References}

1. Lee JK, Coutant C, Kim YC, Qi Y, Theodorescu D, Symmans WF, Baggerly K, Rouzier R and Pusztai L: Prospective comparison of clinical and genomic multivariate predictors of response to neoadjuvant chemotherapy in breast cancer. Clin Cancer Res 16: 711-718, 2010.

2. Rouzier R, Pusztai L, Delaloge S, Gonzalez-Angulo AM, Andre F, Hess KR, Buzdar AU, Garbay JR, Spielmann M, Mathieu MC, Symmans WF, Wagner P, Atallah D, Valero V, Berry DA and Hortobagyi GN: Nomograms to predict pathologic complete response and metastasis-free survival after preoperative chemotherapy for breast cancer. J Clin Oncol 23: 8331-8339, 2005.

3. Kaufmann M, von Minckwitz G, Mamounas EP, Cameron D, Carey LA, Cristofanilli M, Denkert C, Eiermann W, Gnant M, Harris JR, Karn T, Liedtke C, Mauri D, Rouzier R, Ruckhaeberle E, Semiglazov V, Symmans WF, Tutt A and Pusztai L: Recommendations from an international consensus conference on the current status and future of neoadjuvant systemic therapy in primary breast cancer. Ann Surg Oncol 19: 1508-1516, 2012.
4. U.S. Department of Health and Human Services, Food and Drug Administration, Center for Drug Evaluation and Research (CDER): Guidance for Industry Pathological Complete Response in Neoadjuvant Treatment of High-Risk Early-Stage Breast Cancer: Use as an Endpoint to Support Accelerated Approval. Retrieved from: http://www.fda.gov/downloads/Drugs/ Guidance ComplianceRegulatoryInformation/Guidances/ UCM305501.pdf. Accessed on April 10, 2014.

5. Gianni L, Eiermann W, Semiglazov V, Manikhas A, Lluch A, Tjulandin S, Zambetti M, Vazquez F, Byakhow M, Lichinitser M, Climent MA, Ciruelos E, Ojeda B, Mansutti M, Bozhok A, Baronio R, Feyereislova A, Barton C, Valagussa P and Baselga J: Neoadjuvant chemotherapy with trastuzumab followed by adjuvant trastuzumab versus neoadjuvant chemotherapy alone, in patients with HER2-positive locally advanced breast cancer (the NOAH trial): A randomised controlled superiority trial with a parallel HER2-negative cohort. Lancet 375: 377-384, 2010.

6. Hutcheon AW, Heys SD and Sarkar TK; Aberdeen Breast Group: Neoadjuvant docetaxel in locally advanced breast cancer. Breast Cancer Res Treat 79 (Suppl 1): S19-S24, 2003.

7. Untch M, Möbus V, Kuhn W, Muck BR, Thomssen C, Bauerfeind I, Harbeck N, Werner C, Lebeau A, Schneeweiss A, Kahlert S, von Koch F, Petry KU, Wallwiener D, Kreienberg R, Albert US, Lück HJ, Hinke A, Jänicke F and Konecny GE: Intensive dose-dense compared with conventionally scheduled preoperative chemotherapy for high-risk primary breast cancer. J Clin Oncol 27: 2938-2945, 2009.

8. Kuerer HM, Newman LA, Smith TL, Ames FC, Hunt KK, Dhingra K, Theriault RL, Singh G, Binkley SM, Sneige N, Buchholz TA, Ross MI, McNeese MD, Buzdar AU, Hortobagyi GN and Singletary SE: Clinical course of breast cancer patients with complete pathologic primary tumor and axillary lymph node response to doxorubicin-based neoadjuvant chemotherapy. J Clin Oncol 17: 460-469, 1999.

9. Rouzier R, Extra J-M, Klijanienko J, Falcou MC, Asselain B, Vincent-Salomon A, Vielh P and Bourstyn E: Incidence and prognostic significance of complete axillary downstaging after primary chemotherapy in breast cancer patients with T1 to T3 tumors and cytologically proven axillary metastatic lymph nodes. J Clin Oncol 20: 1304-1310, 2002.

10. Esserman LJ, Berry DA, DeMichele A, Carey L, Davis SE, Buxton M, Hudis C, Gray JW, Perou C, Yau C, Livasy C, Krontiras H, Montgomery L, Tripathy D, Lehman C, Liu MC, Olopade OI, Rugo HS, Carpenter JT, Dressler L, Chhieng D, Singh B, Mies C, Rabban J, Chen YY, Giri D, van't Veer L and Hylton N: Pathologic complete response predicts recurrence-free survival more effectively by cancer subset: Results from the I-SPY 1 TRIAL-CALGB 150007/150012, ACRIN 6657. J Clin Oncol 30: 3242-3249, 2012.

11. Mazouni C, Peintinger F, Wan-Kau S, Andre F, Gonzalez-Angulo AM, Symmans WF, Meric-Bernstam F, Valero V, Hortobagyi GN and Pusztai L: Residual ductal carcinoma in situ in patients with complete eradication of invasive breast cancer after neoadjuvant chemotherapy does not adversely affect patient outcome. J Clin Oncol 25: 2650-2655, 2007.

12. von Minckwitz G, Untch M, Blohmer JU, Costa SD, Eidtmann H, Fasching PA, Gerber B, Eiermann W, Hilfrich J, Huober J, Jackisch C, Kaufmann M, Konecny GE, Denkert C, Nekljudova V, Mehta K and Loibl S: Definition and impact of pathologic complete response on prognosis after neoadjuvant chemotherapy in various intrinsic breast cancer subtypes. J Clin Oncol 30: 1796-1804, 2012.

13. Montagna E, Bagnardi V, Rotmensz N, Viale G, Pruneri G, Veronesi P, Cancello G, Balduzzi A, Dellapasqua S, Cardillo A, Luini A, Zurrida S, Gentilini O, Mastropasqua MG, Bottiglieri L, Iorfida M, Goldhirsch A and Colleoni M: Pathological complete response after preoperative systemic therapy and outcome: Relevance of clinical and biologic baseline features. Breast Cancer Res Treat 124: 689-699, 2010.

14. von Minckwitz G, Kaufmann M, Kuemmel S, Fasching PA, Eiermann W, Blohmer JU, Costa SD, Hilfrich J, Jackisch C, Gerber B, Du Bois A, Huober JB, Hanusch CA, Konecny GE, Fett W, Stickeler E, Harbeck N, Mehta K, Loibl S, Untch M and GBG and AGO-B study groups: Correlation of various pathologic complete response (pCR) definitions with long-term outcome and the prognostic value of $\mathrm{pCR}$ in various breast cancer subtypes: Results from the German neoadjuvant meta-analysis. J Clin Oncol 29: 1028, 2011. 
15. Caudle AS, Gonzalez-Angulo AM, Hunt KK, Liu P, Pusztai L, Symmans WF, Kuerer HM, Mittendorf EA, Hortobagyi GN and Meric-Bernstam F: Predictors of tumor progression during neoadjuvant chemotherapy in breast cancer. J Clin Oncol 28: 1821-1828, 2010

16. Boughey JC, Peintinger F, Meric-Bernstam F, Perry AC, Hunt KK, Babiera GV, Singletary SE, Bedrosian I, Lucci A, Buzdar AU, Pusztai L and Kuerer HM: Impact of preoperative versus postoperative chemotherapy on the extent and number of surgical procedures in patients treated in randomized clinical trials for breast cancer. Ann Surg 244: 464-470, 2006.

17. van Nes JG, Putter H, Julien JP, Tubiana-Hulin M, van de Vijver M, Bogaerts J, de Vos M and van de Velde CJ; Cooperating Investigators of the EORTC: Preoperative chemotherapy is safe in early breast cancer, even after 10 years of follow-up; clinical and translational results from the EORTC trial 10902. Breast Cancer Res Treat 115: 101-113, 2009.

18. Zhang GC, Zhang YF, Xu FP, Qian XK, Guo ZB, Ren CY and Yao M: Axillary lymph node status, adjusted for pathologic complete response in breast and axilla after neoadjuvant chemotherapy, predicts differential disease-free survival in breast cancer. Curr Oncol 20: e180-e192, 2013.
19. Ellis MJ, Tao Y, Luo J, A'Hern R, Evans DB, Bhatnagar AS, Chaudri Ross HA, von Kameke A, Miller WR, Smith I, Eiermann W and Dowsett M: Outcome prediction for estrogen receptor-positive breast cancer based on postneoadjuvant endocrine therapy tumor characteristics. J Natl Cancer Inst 100: 1380-1388, 2008.

20. Gonzalez-Angulo AM, McGuire SE, Buchholz TA, Tucker SL, Kuerer HM, Rouzier R, Kau SW, Huang EH, Morandi P, Ocana A, Cristofanilli M, Valero V, Buzdar AU and Hortobagyi GN: Factors predictive of distant metastases in patients with breast cancer who have a pathologic complete response after neoadjuvant chemotherapy. J Clin Oncol 23: 7098-7104, 2005.

21. Tanioka M, Shimizu C, Yonemori K, Yoshimura K, Tamura K, Kouno T, Ando M, Katsumata N, Tsuda H, Kinoshita T and Fujiwara Y: Predictors of recurrence in breast cancer patients with a pathologic complete response after neoadjuvant chemotherapy. Br J Cancer 103: 297-302, 2010. 